\title{
Understanding the genesis of form in Brazilian informal settlements: modelling the environment as a preliminary approach to design
}

\section{SIGRADI2018 TECHNOPOLITICAS}

xxii congresso da sociedade iberoamericana de gráfica digital 22th conference of the iberoamerican society of digital graphics $07|08| 09$ |novembro|2018 iau usp | são carlos | sp br

\author{
Debora Verniz \\ Pennsylvania State University | United States of America | dvp6@psu.edu \\ Danielle Oprean \\ University of Missouri | United States of America | opreand@missouri.edu \\ José Duarte \\ Pennsylvania State University | United States of America | jxp400@psu.edu
}

\begin{abstract}
This work is part of a larger research that proposes an alternative approach to housing for low-income people, based on the model of Brazilian favelas, with the goal of generating housing solutions that are more adapted to physical conditions, affordable, and spatially rich. This paper presents how the favela Santa Marta (Rio de Janeiro, Brazil) was modeled through three types of models: digital, immersive and computational/ analytic model. The argument is that by using a more holistic modelling, it is possible to gain a deeper understanding of the genesis and formal structure of informal settlements and develop better approaches for tackling related challenges.
\end{abstract}

Keywords: Informal settlements; Santa Marta; modelling the environment; shape grammar; virtual environment.

\section{INTRODUCTION}

The world faces a fast population growth coupled with a fast urbanization process. Lacking proper accommodation, part of this growing population resort to self-construction. It is estimated that almost one billion people are living in informal settlements around the world. (United Nations Human Settlements Programme, 2015) Informal settlements are distinguished by poor quality housing, poverty, lack of public and private services, and low integration with the broader community and its opportunities. (United Nations Human Settlements Programme, 2003)

This paper is part of a larger research that aims to propose an alternative approach to housing for lowincome people, based on the model of Brazilian favelas. The goal is to generate housing solutions that are more adapted to physical conditions, affordable and spatially rich. To reach this goal, the research uses as a case study the favela Santa Marta in Rio, Brazil. The research encompasses the following steps: (1) developing different models of representation of Santa Marta, such as digital and immersive virtual models, (2) inferring the Santa Marta shape grammar to create a grammar-based computational model, (3) using the digital and virtual models of Santa Marta in a workshop with specialists to plan an expansion of the favela, and (4) extracting rules for urban design based on the workshop results.

This paper is focused on steps 1 and 2 of the main research. The goal is to model Santa Marta, showing its growth trajectory, which follows a more adaptive approach than traditional urban planning.

\section{STATE OF ART}

The emergence of informal settlements is closely related to rapid city growth. They were first identified in the midnineteenth century in France, America and India. These informal settlements were recognized as a worldwide phenomenon characterized by illegal occupation. (Davis, 2006) In Brazil, the process of informal settlements growth began between the late nineteenth and early twentieth centuries, driven by various factors in each region of the country. (Compans, 2007)

Informal settlements in Brazil are typically called favelas. Favelas usually comprise of large territorial extensions and are characterized by unfinished houses, high density, lack of green areas, urban mobility restrictions, and lack of public facilities intended for health, leisure, sports, and culture (Prefeitura de São Paulo, 2015, United Nations Human Settlements Programme, 2003). Although political policies tried to eradicate favelas during most of the twentieth century, these places house a cheap and available labor force.

This is the main reason for having favelas within proximity to prime areas of the city. (Gonçalves, 2013) Thus, the apparently 'spontaneous and disorderly' development of slums reflect a very clear socioeconomic strategy used by favela residents. (Meirelles \& Athayde, 2014) 


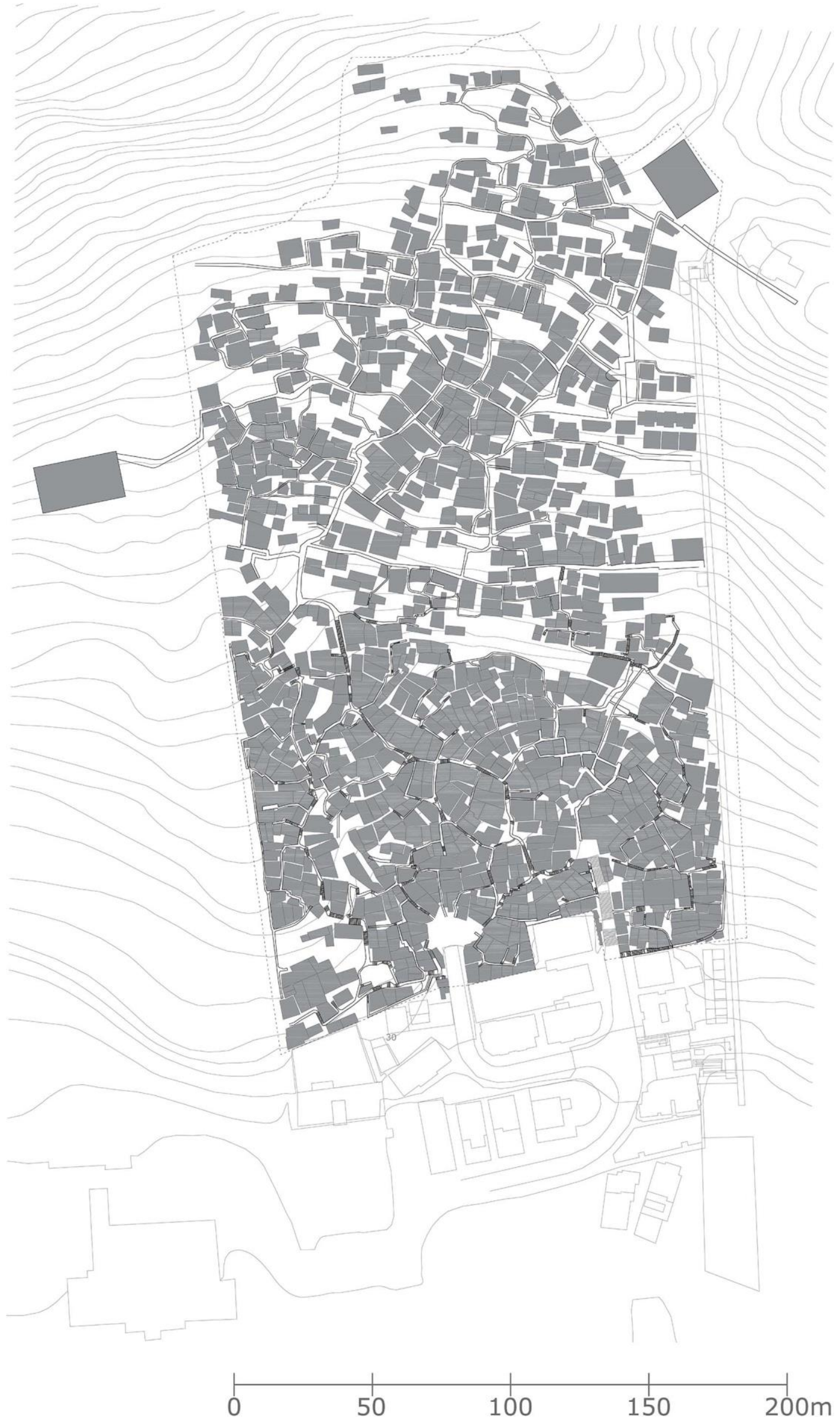

Figure 1: 2D plan of Santa Marta. Source: Verniz and Duarte (2017). 
Mahabir et al. (2016) pointed out how work on informal settlements are focused on different lines of inquiry that have their own set of assumptions and interpretation. The reasons that foment the appearance and growth of informal settlements are complex and require interdisciplinary studies to develop a deep understanding of the phenomenon. As such, these places should be examined using more holistic and systematic approaches. (Mahabir et al., 2016)

Previous publications on the current research have focused partial aspects of the modelling of Santa Marta: Verniz et al. (2016) presents the process of 3D survey to digitally reconstruct the place using a photogrammetry technique; Verniz and Duarte (2017) presents a preliminary version of the analytic, computational model of Santa Marta, a shape grammar that explains its settlement process; and Oprean et ali. (2018) presents the virtual environment used for remote site visiting Santa Marta.

This paper presents how the construction of three models of representation (digital, virtual, and computational) helped to construct a more holistic representation of favela Santa Marta.

\section{METHODOLOGY}

The methodology of this work encompasses the two first steps of the larger research mentioned above. The first step is to develop different models of representation of Santa Marta, which includes the digital and the immersive models. The second step is to infer a shape grammar, called the computational model, that explains the settlement process of Santa Marta. The development of each model involves several sub-steps that are presented in the following subsections.

Data was collected from the place and local archives in three moments: September 2015, June 2016, and May 2017. In September 2015, digital images were taken on site and topographic charts were obtained from the town hall. In June 2016, wide-angle sequential images, $360^{\circ}$ images and videos, and a map of Santa Marta were gathered. In May 2017, additional digital images, 360 images and videos, and audio recordings were added to the collected data. These were then used to generate the models of representation of the case study mentioned above.

To route through the favela used to collect images in the first moment was intuitive: we entered the site at the bottom and then went up as we felt safer, preferably walking through wider pathways and no dead-end routes. In the second and third moments, we planned the route more consciously, considering aspects like accessibility and safety and choosing wider or more continuous pathways. In some cases, the route was corrected on site to guarantee we proceeded through streets with more people, perceived as safer. In all cases we took pictures from points that could be seen from the previous point and filmed the pathway in between.

The information gathered from archives at the Municipality of Rio de Janeiro and at a company called EMOP (Empresa de Obras Publicas) include a topographic chart of the area and a map of the settlement, which were used to generate an updated $2 \mathrm{D}$ plan from the area that contained both topographic and urban information on Santa Marta (Figure 1).

This 2D plan served as a basis to create a digital model of Santa Marta. Together, the 2D plan and the 3D models were used, first to understand the formal structure of the existing settlement, including how buildings related to each other and to the underlying topography, and then how the urban form might have been originated. This analytical, computational model was developed and captured into a shape grammar.

\section{DIGITAL MODEL}

Digital models are utilized to create, represent, analyze, predict, and correct mistakes in urbanism, architecture, and design. When associated with generative tools, it is possible to create a variety of solutions in a relatively short time. Researchers (Klerk \& Beirão, 2017; Santos \& Beirão, 2017) have explored the use of digital models to analyze, represent and even generate architecture and urban models and associated data.

In this work, the digital model is a volumetric representation of Santa Marta that includes both the sloped terrain and the buildings. The digital model is important to provide a tridimensional visualization of the settlement and inspecting the relation between buildings and terrain.

To create the digital model, the 2D plan from Santa Marta collected from the town hall was updated with information gathered on site and recorded in images and videos. The height of buildings was estimated in some cases considering the number of floors and a floor height of 3 meters, the standard in Brazil. The number of floors for 282 buildings, $26.5 \%$ of the total, was identified through visual analysis of images. To calculate the number of

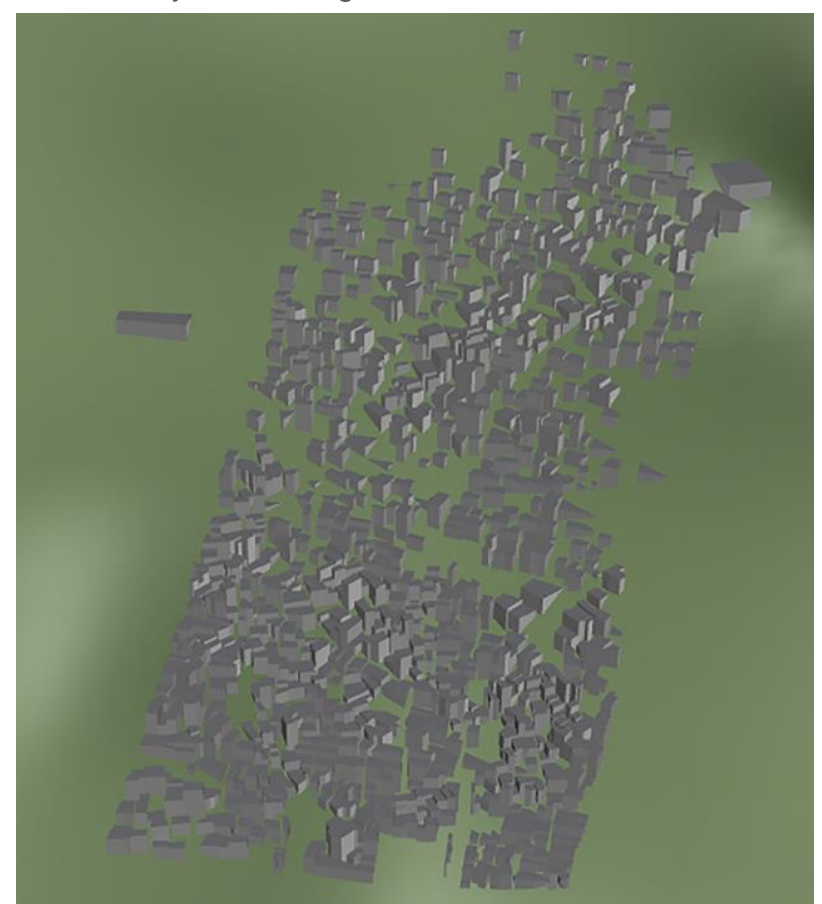

Figure 2: Digital model representing Santa Marta's buildings. Source: Authors.

floors of the remaining buildings, we considered the percentage of buildings with a specific number of floors in 
the known portion and randomized heights for a similar percentage in the unknown one.

The terrain surface was generated using information from the contour lines and the command "Surface>Path", from Rhinoceros. To generate the building volumes, we created a grasshopper code to project the building footprints onto the terrain surface, then extruded the buildings with the known and randomized heights. The resulting digital model is shown in Figure 2 and Figure 3.

The digital model was used as input for milling a physical model representing the volumetric aspect of Santa Marta, which was crucial to get a more tangible view of the favela and gain a deeper understanding of its form.

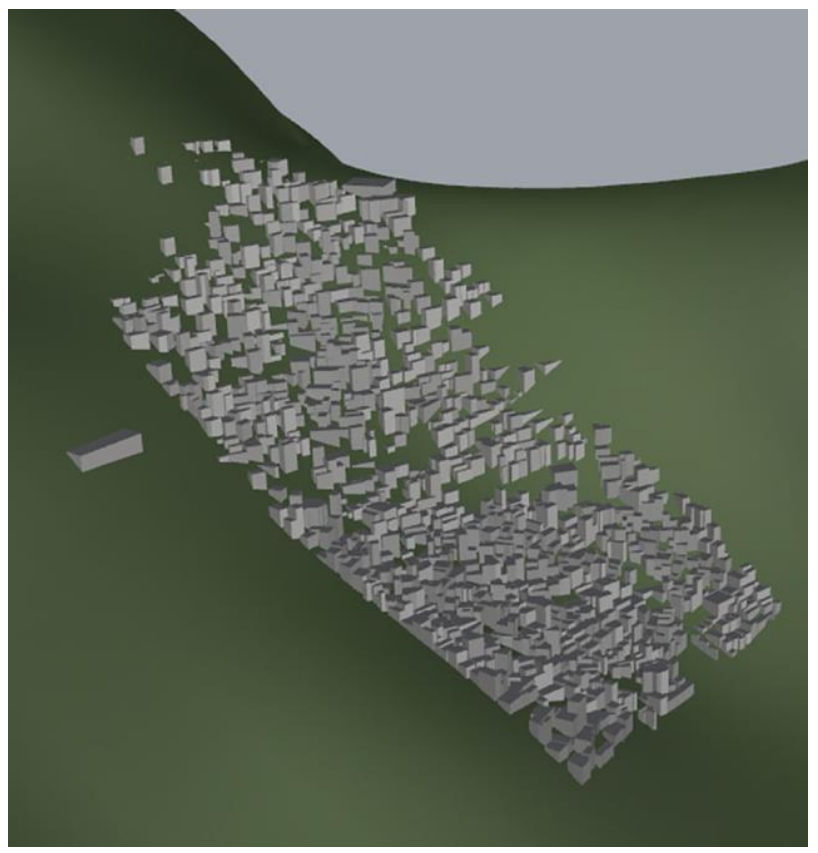

Figure 3: Digital model representing Santa Marta's buildings. Source: Authors.

\section{IMMERSIVE MODEL}

The immersive model presented a first-person, humanscale, perspective of Santa Marta, portraying key pathways and prominent features. The immersive model comprised of $360^{\circ}$ images and videos and a 2D plan of the settlement. The immersive model was portrayed across three different immersive technology mediums: WebVR, a mobile application, and an HTC Vive application (Figure 4). The model was the same, normalized, across all mediums by the source material quality, using a Ricoh Theta S camera. All content was aligned with paths on the 2D plan. This allowed for the subtle differences between each technology medium to be examined for realism.

The immersive model was created to account for visual realism as a factor of fidelity (quality of resolution and congruity) (Mania et al., 2006). For visual fidelity, $360^{\circ}$ content was used as opposed to generating 3D models. This distinction is necessary as $360^{\circ}$ imagery contains more realism compared to $3 \mathrm{D}$ models, capturing many more details. While 3D models may address the shape of objects better, they would be highly intensive to create with the vast amount of details prominent in the settlement. Additionally, $360^{\circ}$ imagery provides an egocentric perspective that wraps around a user, a feature available through several viewing mediums.

Each medium enabled a viewing (interaction) fidelity representative of key affordances provided by each, the interaction was the same but the way to access that interaction varied by technology. All three mediums allowed for navigation of the $360^{\circ}$ content by a rotational method, the method of accessing this rotational agency is what differentiated each. The WebVR allowed for mouse or device gyroscope to navigate with mouse-based pointand-click functionality. The mobile app, viewable through a cardboard headset, used the device gyroscope to allow for interaction (point-and-click by tapping the screen with the cardboard button) and viewing. Lastly, the HTC Vive used head-tracking with point-and-click functionality attached to the hand-controller. Figure 4 illustrates the Immersive model of Santa Marta being used through a $360^{\circ}$ imagery across its three mediums.

\section{ANALYTIC MODEL}

The Santa Marta shape grammar is an analytic model that explains the spontaneous settlement process of the territory by the favela. This analytic model is computational in the sense that it computes the complex existing form from simple initial shapes.

In the development of the analytical model, we have identified three factors that seem to influence the process: topography, urban context, and functional organization of the buildings. These factors play an important role on how the buildings are located, shaped, and aligned. (Verniz and Duarte, 2017)

Steep topography is an important feature of Rio de Janeiro, visible in the skyline. The hill range forms a barrier to the formal city, which avoids steep areas but favelas usually settle on these areas. As buildings tend to align with contour lines, topography influences both the shape of individual buildings and the resulting urban configuration,

The urban context constrains accessibility, construction decisions, and the perception of safety in the placement of buildings in favelas. The favela grows from access point from the formal city, with buildings being located in available places that are the closest from such points, sharing walls with existing buildings whenever possible. In places near the favela's borders, which in the case of Santa Marta are clearly delimited by walls, buildings align with the limits rather than the contour lines. 

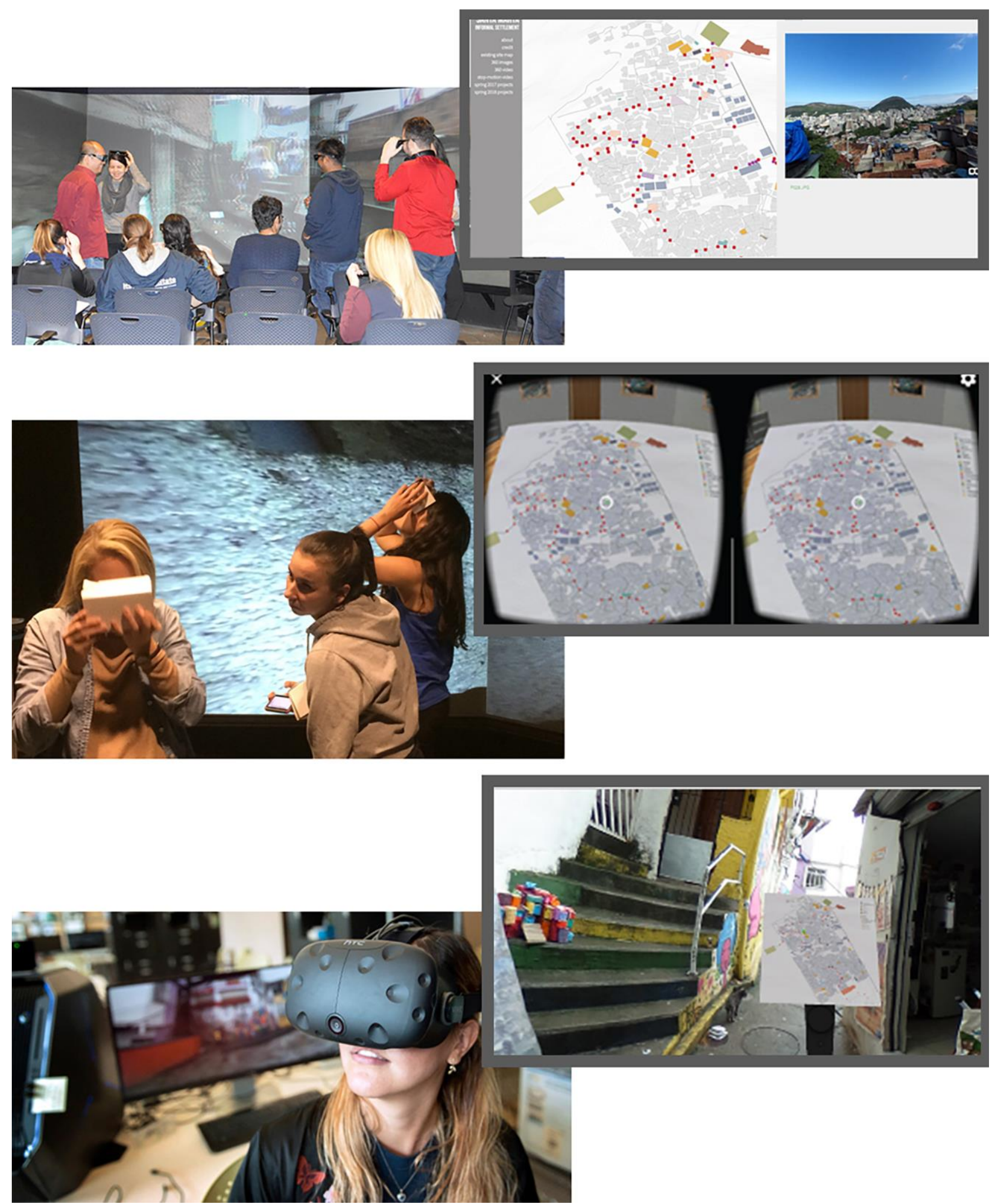

Figure 4: Immersive model of Santa Marta through $360^{\circ}$ imagery across three mediums: top) WebVR, middle) mobile application, bottom) HTC Vive. Source: Authors.

Functional organization of the houses also influences how buildings are shaped and sized. Nearly half of the favela's buildings have a footprint shaped as a four-sided polygon, although they can be up to eleven sided. Our interpretation is that complex footprint shapes can be the result of expansions that occurred over time.

Buildings were classified according to use, which showed that 1,011 buildings of them are used as housing or housing and commerce, which represents $94.7 \%$ of the total. Buildings were further classified by the number of sides of their polygonal footprint, which can vary between four and 11 and their shapes can be concave or convex. There are no two buildings alike. The process of generating a footprint starts with a four-sided polygon representing the basic footprint. All the other shapes can be derived from this basic shape either by adding a vertex or an annex. 


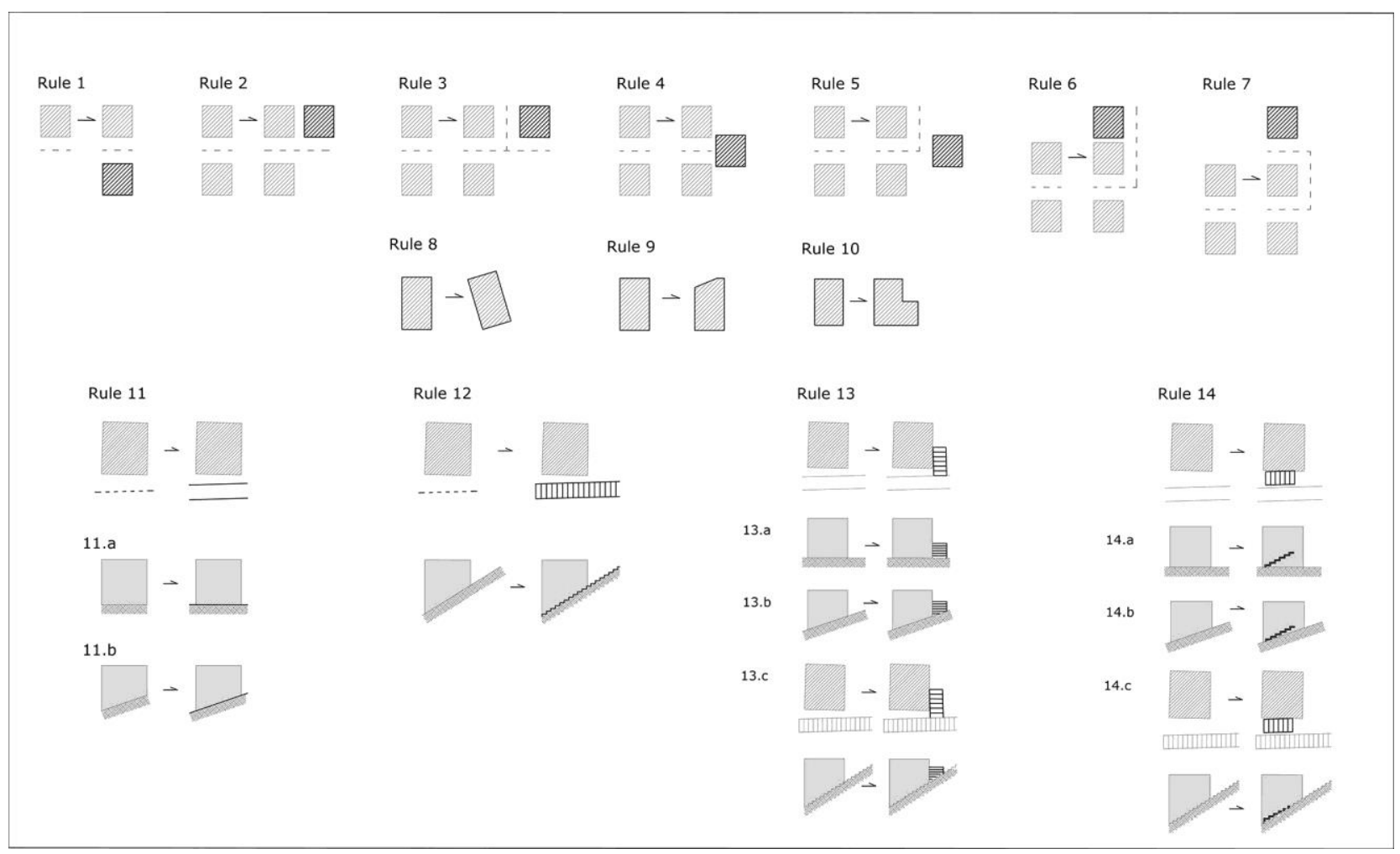

Figure 5: Selected simplified rules of the Santa Marta shape grammar. Source: Authors.

To detail the analysis of buildings, a Grasshopper code was created, which permits to rapidly analyze the shape of the footprint and determine its area, number of sides, internal angles, and side length.

A parametric set of rules was developed to explain the generation of the different building shapes and dimensions. These rules are divided in two subcategories: the first one is related with building placement, alignment and shaping and encompasses Rules 1 to 10; the second subcategory encompasses Rules 11 to 14 and it is related to the placement of circulation. Figure 5 presents a set of simplified rules from the Santa Marta shape grammar.

To validate the model, the same procedure adopted by Duarte et al. (2007) in the development of the grammar for the Marrakesh medina was followed. The grammar was developed using the southern portion of the settlement and tested on the northern portion. Figure 6 shows the derivation of the northern portion using Rules 1 to 14 , validating the grammar.

\section{DISCUSSION}

Several models were generated to represent Santa Marta in its different aspects. The 2D plan constructed with the topographic charts and the settlement's map contains topographic and urban information about the case study. This representation was used as base to construct the models. It organizes the data about the case study and it contributes with the construction of a map for Santa Marta, which was inexistent. As it happens with many
Brazilian favelas, the municipality does not have a complete map for the area.

The digital model is a tri-dimensional representation that was constructed with parametric modelling. It provides a broader visualization of the neighborhood and the hill where Santa Marta is settled, providing the perception of the terrain steepness and building density. It presents a low level of detailing as it is a volumetric representation. This model was used as a basis to understand the favela morphology and develop the analytic model.

The immersive model is a first-person representation of high trafficked areas in the settlement designed to provide views of specific features and characteristics at a human scale. The model was portrayed across three different viewing mediums to identify how the fidelity, degree of realism, of the content improved key details in the digital and analytic models.

The analytic model permits to describe the settlement process of Santa Marta. This model consists in a parametric shape grammar that captures how different forces influence the generation of the built form. These forces are topography, urban context, and functional organization of the buildings. The analytic model explains geometrically (rather than historically) how the settlement process of Santa Marta can occur. This means the goal is not to explain exactly how the form was generated along time but show how different factors can influence the resulting geometry. 

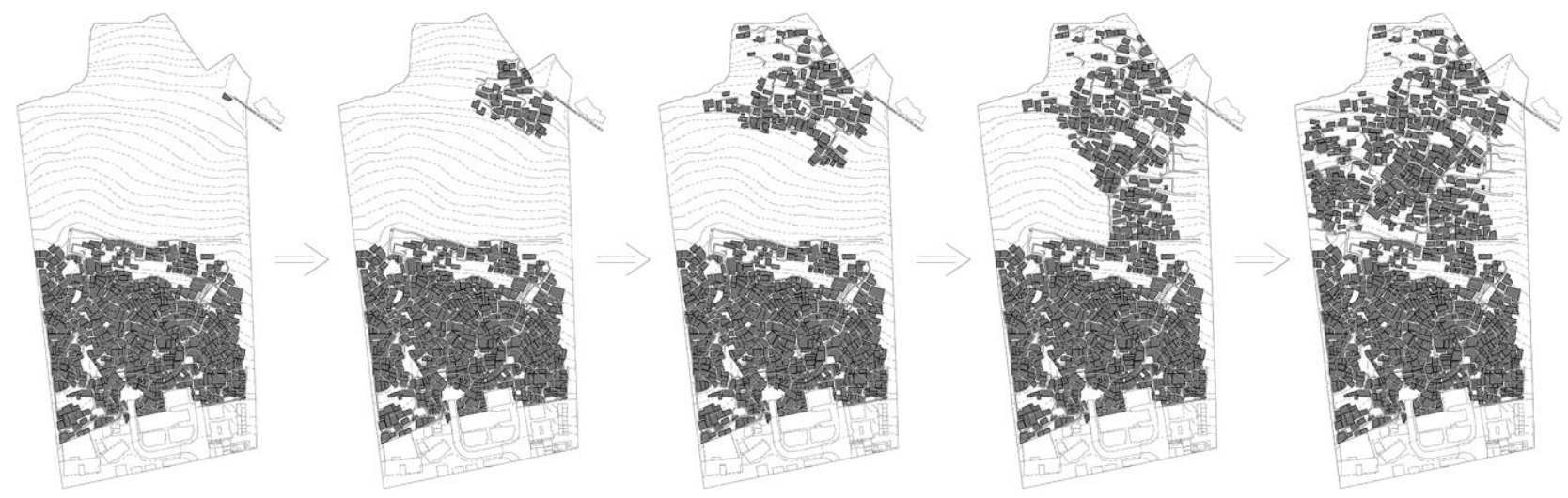

Figure 6: Derivation of the Northern portion of Santa Marta using the proposed shape grammar. Source: Authors.

The models were produced in order to analyze and understand the case study. They are built up on representation tools and were fundamental for the construction of a representational base to continue the research.

\section{CONCLUSIONS}

This paper presents how different models of Santa Marta were developed to represent the existing built environment and help to understand the emergence of informal settlements.

With three distinct models it was possible to provide a diverse representation of the case study with its volumetry, a realistic and immersive representation, and the settlement process' logic. A single model would not present all this information with the same quality or fidelity as each technology has its own strengths and compatibilities.

The digital model represents the volumetric features of Santa Marta and parametric constrains coded in grasshopper enabled to extract various kinds of information from the geometry and characterize the building and urban forms. The immersive model provided a human-scaled perspective to identify the structure of the settlement from the ground-view. The computational model explains the settlement process based on parametric constrains and it is essential to provide a better understanding of the existing settlement.

Although this research uses as a case study a specific favela, the methodology can be generalized to other informal settlements in similar conditions. Future steps on the development of this research concern proposing guidelines to plan expansions of existing favelas using rules that are modified versions of the rules extracted from their analysis. The goal is to plan settlements that share some of the positive features of informal settlements, while avoiding negative ones. We hope this framework may constitute a step towards the development of an alternative approach to existing planning strategies. Although favelas are a result of social and historical problems, they have resisted and grew in number and size for more than a century. Their configurations reflect an economic construction logic that although nonplanned, is visually attractive and emblematic of Rio de Janeiro.
In summary, the ultimate goal of this research is to extract design guidelines for the design of new settlements in contexts similar to those where favelas are usually found. The idea is to produce urban environments that are as affordable and have the same kind of spatial richness as favelas while avoiding many of their inherent flaws.

\section{ACKNOWLEDGMENTS}

This work has been supported by the University of Lisbon and The Pennsylvania State University. The work of the PhD candidate Debora Verniz was funded by grant 207028/2014-1from the Science Without Borders Programme administered by the Brazilian National Council for Science and Technological Development (CNPq) from March, 2015 to February, 2018.

\section{REFERENCES}

Compans, Rose. 2007. "A cidade contra a favela. A nova ameaça ambiental" (The city against the slum. The new environment threat). Revista Brasileira de Estudos Urbanos e Regionais 9 (1): 83-99. Retrieved May 26, 2015 (http://unuhospedagem.com.br/revista/rbeur/index.php/anais/ article/view/3514).

Davis, Mike. 2007. Planet of slums. London: Verso.

Duarte, Jose, João Rocha and Gonçalo Soares. 2007. "Unveiling the structure of Marrakesh Medina: a shape grammar and interpreter for generating urban form". Artificial Intelligence for Engineering Design, Analysis and Manufacturing. Volume 21. Cambridge University Press. 317-349.

Gonçalves, Rafael Soares. 2013. "A política, o direito e as favelas do rio de janeiro: um breve olhar histórico" (The policy, the right, and the favelas in Rio de Janeiro: a brief historical look). URBANA: Revista Eletrônica do Centro Interdisciplinar de Estudos sobre a Cidade, 1 (1): 1-23. $\begin{array}{llll}\text { Retrieved } & \text { March } & 17, & \end{array}$ (http://dx.doi.org/10.20396/urbana.v111.8635115).

Klerk, R. de, \& Beirão, J. N. (2017). CIM-St A design grammar for street cross sections. In A. Fioravanti, S. Cursi, S. Gargaro, G. Loffreda, G. Novembri, \& A. Trento (Eds.), ShoCK! Sharing Computational Knowledge! Proceedings of the 35th eCAADe Conference - Volume 2 (pp. 619-928). Rome.

Mahabir, Ron, Andrew Crooks, Arie Croitoru, Peggy Agouris. 2016. "The study of slums as social and physical constructs: challenges and emerging research opportunities". Regional Studies, Regional Science, 3:1, 399-419. DOI: 10.1080/21681376.2016.1229130

Mania, Katerina, Dave Wooldridge, Matthew Coxon, and Andrew Robinson. 2006. "The Effect of Visual and Interaction Fidelity on Spatial Cognition in Immersive Virtual Environments." 
IEEE transactions on visualization and computer graphics 12 (3): 396-404. doi:10.1109/TVCG.2006.55.

Meirelles, Renato and Celso Athayde. 2014. Um país chamado favela. A maior pesquisa já feita sobre a favela brasileira. São Paulo: Editora Gente.

Oprean, Danielle, Debora Verniz, Jiayan Zhao, Jan Oliver Wallgrün and José Duarte. 2018. "Remote studio site experiences: investigating the potential to develop the immersive site visit". Fukuda, W. Huang, P. Janssen, K. Crolla, S. Alhadidi (eds.), Learning, Adapting and Prototyping. Proceedings of the 23rd International Conference of the Association for Computer-Aided Architectural Design Research in Asia (CAADRIA) 2018, Volume 1, 421-430.

Prefeitura de São Paulo. 2015. Cadernos de formação: direito à cidade (Training books: right to the city). São Paulo: Editora Instituto Paulo Freire.

Santos, D. M., \& Beirão, J. N. (2017). Generative tool to support architectural design decision of earthbag building domes. In SIGraDi 2017, XXI Congreso de la Sociedad Iberoamericana de Gráfica Digital (pp. 538-543). São Paulo: Blucher. https://doi.org/10.5151/sigradi2017-083
United Nations Human Settlement Programme. 2015. Practical guide to designing, planning and implementing citywide slum upgrading program. Nairobi: UNON.

United Nations Human Settlement Programme. 2003. The challenge of slums: global report on human settlements. London; Sterling, VA: Earthscan Publications.

Verniz, Debora and José Duarte. 2017. Santa Marta Urban Grammar - Towards an understanding of the genesis of form Fioravanti, A, Cursi, S, Elahmar, S, Gargaro, S, Loffreda, G, Novembri, G, Trento, A (eds.), ShoCK! - Sharing Computational Knowledge! - Proceedings of the 35th eCAADe Conference - Volume 2, Sapienza University of Rome, Rome, Italy, 20-22 September 2017, pp. 477-484 http://papers.cumincad.org/cgibin/works/Show?ecaade2017_264

Verniz, Debora, Luis Mateus, José Duarte and Victor Ferreira. 2016. "3D Reconstruction Survey of Complex Informal Settlements - Towards an understanding of the genesis of form". Herneoja, Aulikki; Toni Österlund and Piia Markkanen (eds.), Complexity \& Simplicity - Proceedings of the 34th eCAADe Conference - Volume 2, University of Oulu, Oulu, Finland, 22-26 August 2016, pp. 365-370 http://papers.cumincad.org/cgibin/works/Show?ecaade2016_110 